Caries Res. 1983;17(Suppl. 1):I-IV

\title{
Contents, Vol. 17, Supplement 1, 1983
}

\section{Contents}

Preface

Gron, P.; Ericsson, Y 1

Inorganic Chemistry

Monofluorophosphate - Its Beginning

White, W.E 2

Fluoride and Monofluorophosphate Analysis

Lindahl,C.B 9

Discussion: Schreiber, R.S 16

Biochemistry in General

Biochemistry of Monofluorophosphate

Pearce, E.I.F 21

Discussion: Ericsson, $\mathrm{Y} \quad 34$

Toxicity

Toxicology of Monofluorophosphate

Smith, F.A.; Hodge, H.C 36

Discussion: Lim, J.K 42

Physiology

Monofluorophosphate Physiology: General Considerations

Ericsson, Y 46

Monofluorophosphate Physiology: The Effects of Fluoride on Bone

Baylink, D.J.; Duane, P.B.; Farley, S.M.; Farley, J.R 56

Discussion: Whitford, G.M.; Pashley, D.H.; Allison, N.B 69

Reaction of Monofluorophosphate with Apatitic Substrates

Duff, E.J 77

Discussion: Rooij, J.F. de; Arends, J 88

Interaction of Monofluorophosphate with Plaque and Saliva

Rølla,G 91

Discussion: Kashket, S 96

IV Contents

Utilization in Oral Preparations

Monofluorophosphate Utilization in Oral Preparations: Laboratory Observations

Mellberg,J.R 102

Discussion: Feller, R.P

Clinical Studies of Monofluorophosphate Dentifrices

DePaola,P.F 119

Discussion: Horowitz, H.S 130

Concluding Remarks

Gron, P.; Ericsson, Y 136 
Author Index 137 